\title{
Assessment of prognosis in chronic coronary artery disease
}

\section{T M Bateman, E Prvulovich}

or many patients with chronic coronary artery disease, risk stratification as to likelihood of cardiac death lays at the basis of choosing between the two major therapeutic options of medical management or revascularisation. The target population is those with an intermediate risk of cardiac death, as patients known to be at high or low risk are already adequately risk stratified for clinical decisions. Perfusion imaging is frequently used for these purposes because it can separate patients into low $(<1 \%)$, intermediate $(1-3 \%)$, and high $(>3 \%)$ likelihoods for the major coronary events. In general, contemplation for revascularisation therapy for patients with mild to moderate symptoms would depend on the likelihood of a major coronary event being greater than $3 \%$ per year, in whom revascularisation may confer a survival advantage. Someone with a $<1 \%$ annual event rate might best be managed medically, as the mortality for patients undergoing revascularisation procedures is at least $1 \%$. Management of patients whose perceived risk of a major coronary event is in the range of $1-3 \%$ annually will be individualised, considering such factors as age, compliance with important medications known to reduce risk (such as statins, angiotensin converting enzyme (ACE) inhibitors, $\beta$ blockers), and willingness to undergo periodic follow up.

\section{NUCLEAR CORRELATES OF PROGNOSIS}

Perfusion imaging has value in risk assessment because it permits quantitative assessment of stress induced myocardial perfusion and, if ECG gated single photon emission computed tomography (SPECT) is used, left ventricular function. In patients with known or suspected coronary artery disease a normal perfusion scan is very valuable because it indicates a benign prognosis. Between 1994 and 2001, 16 different studies, including approximately 21000 patients with a normal perfusion study (fig 1) and mean follow up of 28 months, were reported ${ }^{1}$; the rate of cardiac death or of myocardial infarction was less than $1 \%$ per year (table 1), a rate similar to that of an asymptomatic population. Thus, whether minor coronary artery disease is present or not, further investigation can be avoided. Importantly, the negative predictive value of perfusion imaging is independent of the radiotracer used, method of stress, imaging technique, and population studied. Over the same time period 13 studies comparing outcomes of patients with abnormal and normal perfusion studies, including 16000 patients and a mean follow up period of 28 months, reported an average ninefold higher event rate $(6.7 \%$ annually) in patients with abnormal studies ${ }^{1}$ (table 2 ).

The most important variables that predict the likelihood of future events are the extent and severity of inducible perfusion abnormality. ${ }^{2}$ Although both patients depicted in figs 2 and 3 have stress induced perfusion defects, the extent and severity of the defect in fig 3 is much larger, correlating with a higher likelihood of a major coronary event. Other predictors of high risk are: (1) increased lung thallium uptake, ${ }^{3}$ which indicates raised pulmonary capillary pressure; (2) ventricular dilation that is greater in stress images than at rest $^{4}$; (3) the extent of myocardial infarction; (4) left ventricular ejection fraction (LVEF). ${ }^{5}$ Generally speaking, the more abnormal the scan, the higher the likelihood of future coronary events. Extensive data from multiple centres, using all three common radiotracers, clearly establishes that the high risk patient is one with extensive perfusion defects and reduced LVEF, that a patient with no, or minimal, perfusion defects is low risk, and those with limited size perfusion defects are at intermediate risk.

An important evolving understanding is that the nuclear data may provide information which enables differentiation of patients at risk of cardiac death from those at risk of nonfatal myocardial infarction. ${ }^{67}$ Markers of left ventricular function such as LVEF, the extent of myocardial infarction, and increased lung thallium uptake tend to predict cardiac mortality, while ischaemic manifestations such as extent of inducible perfusion abnormality, stress induced regional wall motion abnormalities, or a drop in LVEF are better predictors for acute coronary syndromes.

Given that the majority of myocardial infarcts occur at the site of unstable but mild coronary stenoses ${ }^{8}$ which remain undetected by perfusion imaging, the strong prognostic value of the nuclear technique warrants explanation. One possibility is that limited perfusion reserve caused by abnormal endothelial function is an indirect marker of plaque instability; another is that most patients with extensive mild plaques also have more severe stenoses detectable by perfusion studies. Whichever is the correct explanation, it is clear that perfusion imaging identifies the patient at risk rather than the lesion likely to rupture.

\section{INCREMENTAL VALUE OF PERFUSION IMAGING}

Numerous studies have demonstrated the incremental value of perfusion imaging for risk stratification after knowledge of patient characteristics such as sex, age, and symptoms. ${ }^{9}{ }^{10}$ It also provides significant additional prognostic value after exercise electrocardiography, even in patients with a good exercise capacity on treadmill testing and a favourable prognosis. $^{11}{ }^{12}$ Information about left ventricular function derived from the use of ECG gating, now performed in more than $80 \%$ of SPECT studies in the USA and probably $20-30 \%$ of UK studies, increases the amount of prognostic data still further. In one of the earlier studies of ECG gated SPECT for prognosis, Sharir and colleagues ${ }^{13}$ showed that post-stress LVEF and end systolic volume both provided added information over perfusion defect analysis alone, in predicting cardiac death. More recently, attention has focused on the differences in LVEF post-stress versus at rest ${ }^{14}$; the former in some cases can reflect myocardial stunning and may be important data in estimating the severity of obstruction in a coronary territory.

\section{PERFUSION IMAGING AS GATEKEEPER}

The principle of using perfusion imaging as a gatekeeper to coronary angiography has been tested in several studies. Bateman and colleagues ${ }^{15}$ showed that in a private practice setting this was already a practice standard by the early 1990s such that referral to coronary angiography after normal, mild to moderately abnormal, and severely abnormal perfusion scans was $3.5 \%, 9 \%$, and $60 \%$, respectively. Importantly, a policy of selective referral to coronary angiography based upon high risk findings is defensible as patients with mild to 


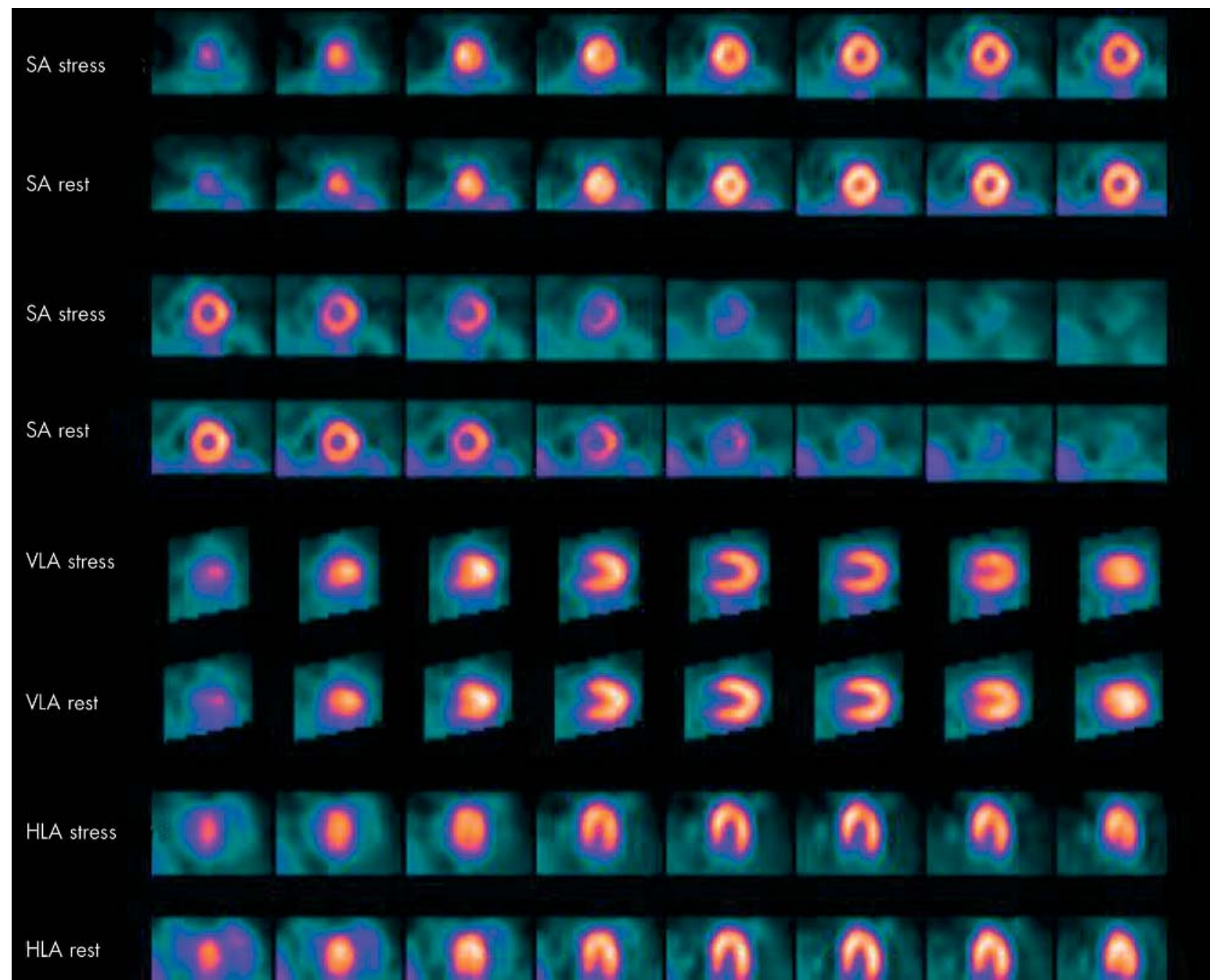

Figure 1 Images acquired after dynamic exercise and following a resting injection of sestamibi. The images are very similar; they show a homogeneous uptake of tracer throughout the myocardium. Hence, there is no coronary obstruction and the likelihood of future coronary events is very low (less than 1\% per year). HLA, horizontal long axis; SA, short axis; VLA, vertical long axis.

moderate abnormalities, managed medically, have outcomes comparable to those undergoing invasive evaluation and subsequent angioplasty.

\section{COST EFFECTIVENESS}

Shaw and colleagues $^{16}$ recently published the results of a multi-centre trial of more than 11000 angina patients recruited from seven different centres. Those who went directly to coronary angiography had a much higher utilisation of subsequent revascularisation, similar rates of death and myocardial infarction, and much higher attendant costs compared to those who were tested first with perfusion imaging and referred to catheterisation dependent on the findings. Hachamovitch ${ }^{6}$ reported $33.5 \%$ cost savings when referral to catheterisation was reserved for patients with

Table 1 Risk of major cardiac events in patients with a normal stress myocardial perfusion SPECT study

\begin{tabular}{lllll}
\hline Years & $\begin{array}{l}\text { Number of } \\
\text { studies }\end{array}$ & $\begin{array}{l}\text { Number of } \\
\text { patients }\end{array}$ & Follow up & $\begin{array}{l}\text { Events per } \\
\text { year }\end{array}$ \\
\hline $1994-2001$ & 16 & 20983 & 28 months & $0.7 \%$ \\
\hline
\end{tabular}

moderate to severely abnormal scans rather than all abnormal scans.

\section{SELECTED SUBSETS}

Although initial prognostic data were acquired for largely male populations aged less than 70 years, recent studies have firmly established that the prognostic power of normal and abnormal myocardial perfusion images is equally strong in men and women. In fact women with severe abnormalities may have a worse outcome than men with severe abnormalities. ${ }^{17}$ Extreme age is also no bar to effective risk stratification by perfusion imaging.

Table 2 Relative risk of major cardiac events in patients with known or suspected coronary artery disease in relation to results of a stress myocardial perfusion study

\begin{tabular}{llll}
\hline Years & $\begin{array}{l}\text { Number of } \\
\text { studies }\end{array}$ & \multicolumn{2}{l}{$\%$ Hard events per year } \\
\cline { 3 - 4 } \cline { 3 - 4 } & Normal & Abnormal \\
\hline $1994-2001$ & 13 & $0-1.3$ & $2.0-14.3$ \\
\hline
\end{tabular}


Table 3 Specific patient subsets in which SPECT myocardial perfusion imaging has been shown to confer useful prognostic information

\begin{tabular}{ll}
\hline Suspected CAD & Post-revascularisation \\
Known CAD & LBBB \\
Men and women & Pacemakers \\
Stable symptoms & LVH \\
Unstable symptoms & Post-infarction \\
Elderly & Before non-cardiac surgery \\
\hline
\end{tabular}

CAD, coronary artery disease; LBBB, left bundle branch block; LVH, left ventricular hypertrophy.

The value of perfusion imaging for risk stratification has been extensively analysed for subsets of patients (table 3) and these will now be considered in turn.

\section{PRESENTATION WITH CHEST PAIN \\ Normal resting ECG}

Patients with chest pain and a normal resting ECG who are able to exercise will often be adequately risk stratified by exercise electrocardiography. Those able to exercise to high workload without chest pain or ST depression and with an adequate blood pressure response to exercise are at low risk. Those with chest pain at low workload associated with ST depression and exercise induced hypotension are at high risk. Intermediate risk results, however, are common-occurring in approximately $30-55 \%$ cases-and it is in these patients that perfusion imaging has a major role. Several publications have shown the incremental prognostic value of perfusion imaging within this risk category and have demonstrated a closer association of subsequent coronary angiographic data with the results of the perfusion scan than with the exercise ECG. One such study is that reported by Gibbons and colleagues. ${ }^{18}$ In a multi-centre study of nearly 5000 patients with an intermediate Duke treadmill score, but normal or near normal perfusion studies, the annual rate of cardiac death or non-fatal myocardial infarction was $0.7 \%$, and only $17 \%$ of patients underwent coronary angiography in the subsequent five years.

\section{Abnormal resting ECG}

Where the resting ECG is abnormal because of left bundle branch block, permanent pacing, pre-excitation, left ventricular hypertrophy or drug effects, exercise electrocardiography can provide limited prognostic data based on exercise duration, but electrocardiographic changes are unhelpful. In contrast, perfusion imaging appears to maintain its prognostic power, ${ }^{19}$ providing vasodilator stress is used to avoid the artefactual perfusion defects associated with dynamic exercise and inotropic stress. ${ }^{20}$

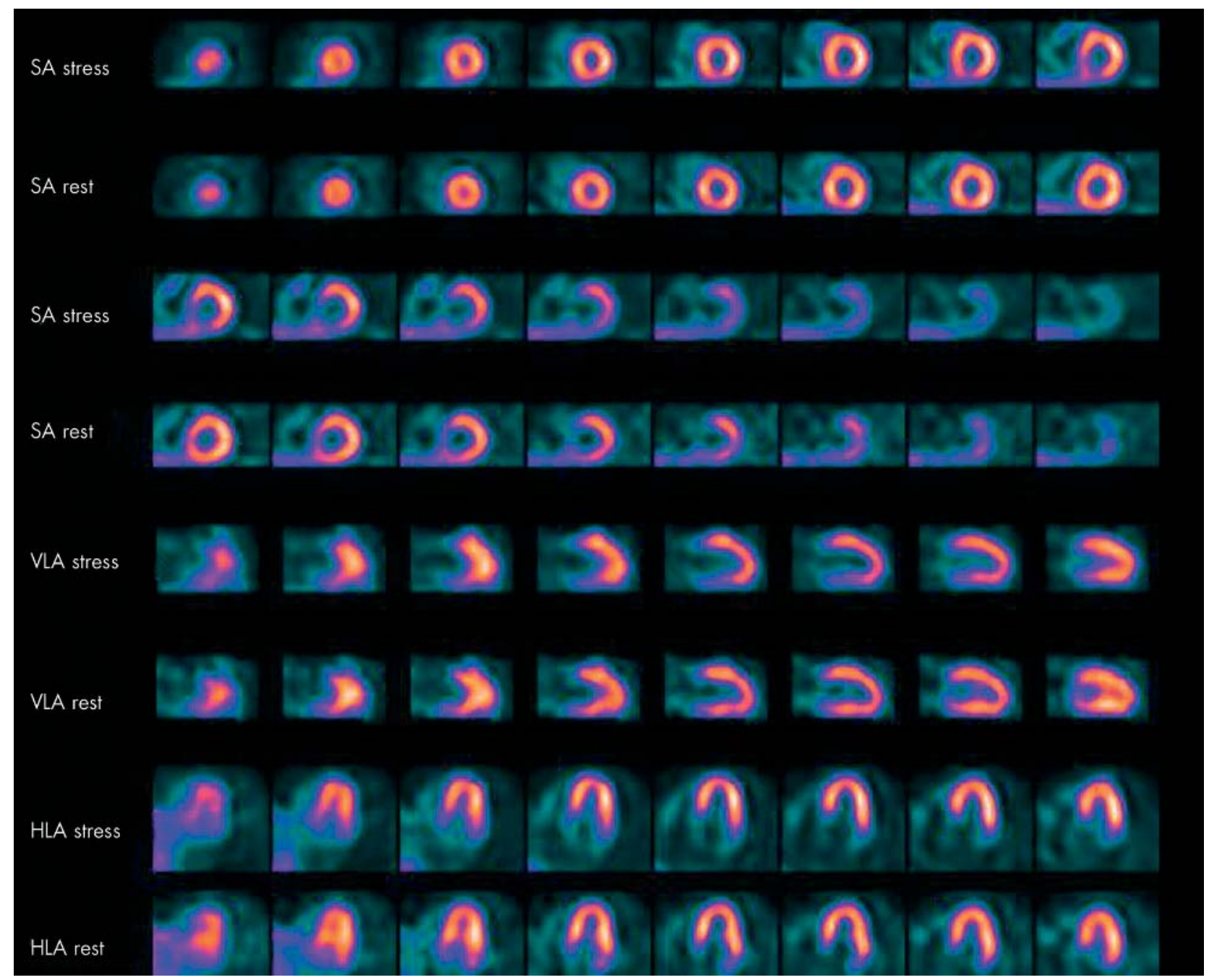

Figure 2 Images showing inferior inducible ischaemia of mild to moderate severity. The scan appearance suggests a low to intermediate risk of future coronary events. Technical details are as for fig 1 . 


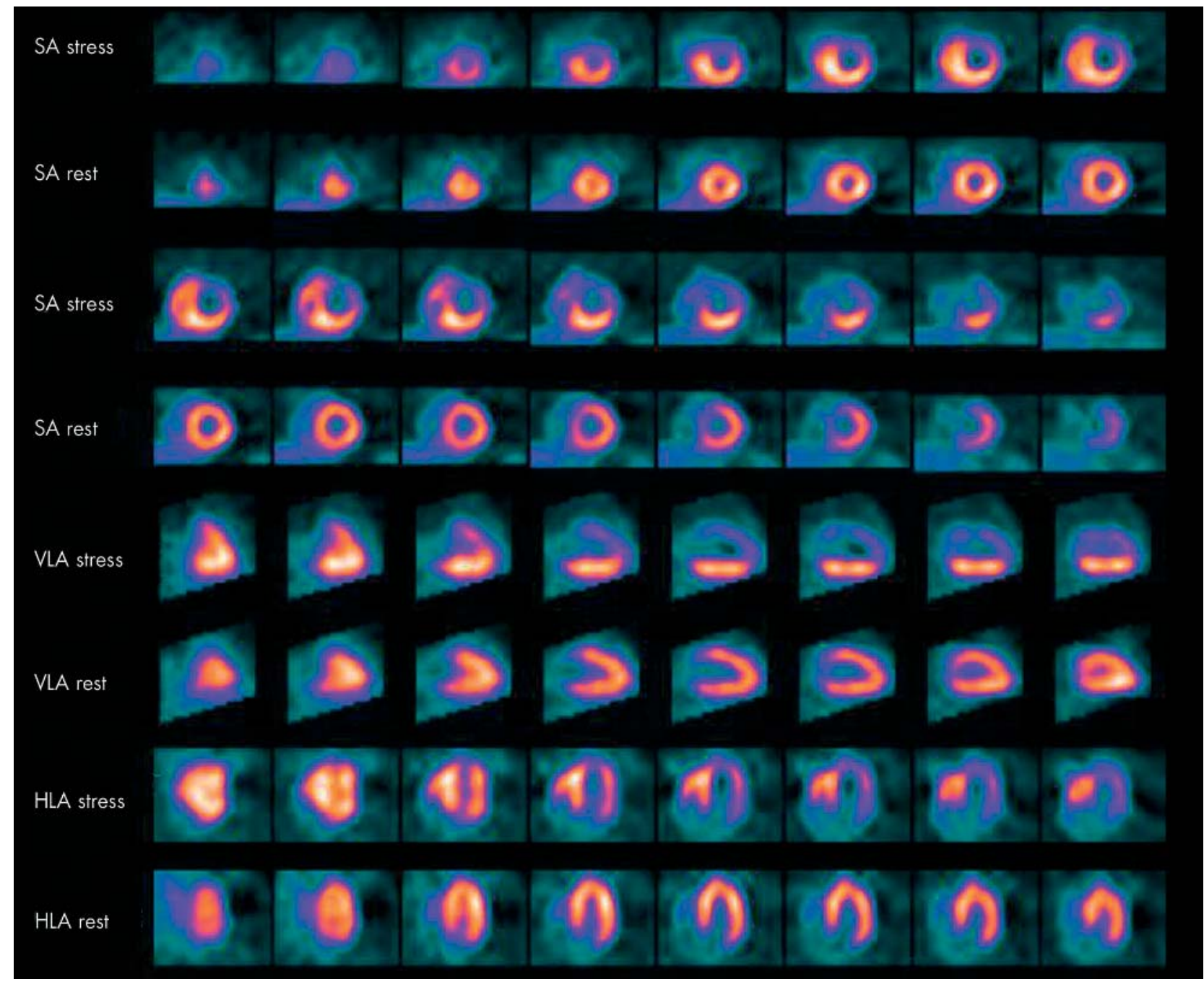

Figure 3 Images showing anteroapical and lateral inducible ischaemia of moderate severity. The scan appearance suggests a high risk of future coronary events. Technical details are as for fig 1.

\section{Patients unable to exercise}

Perfusion imaging after submaximal exercise may underestimate the extent of ischaemia and confer an unduly favourable prognosis. Patients unable to exercise sufficiently to achieve the maximum predicted heart rate for their age and sex should therefore be risk stratified using perfusion imaging coupled with pharmacological stress. The risk of cardiac death in such patients is higher than in patients able to exercise, this is probably caused by the increased underlying risk in this population. ${ }^{21}$

\section{PATIENTS WITH DIABETES}

A number of recent studies indicate that patients with diabetes with symptoms suggestive of coronary artery disease are as effectively risk stratified by perfusion imaging as nondiabetic patients. A normal study predicts low coronary risk in diabetic and non-diabetic patients alike, with the caveat that the period for which a normal scan result can be considered valid may be shorter in diabetic patients, presumably because they develop coronary artery disease at an accelerated rate. This idea of a short warranty period is a relatively new concept and stems from work by Giri and colleagues ${ }^{22}$ who followed 929 diabetic and 3826 non-diabetic patients after perfusion imaging. Survival during the first two years of follow up in patients with a normal perfusion study was similar between the two groups; however, after two years coronary event rates increased in diabetic patients but not in non-diabetic patients, suggesting retesting of diabetic patients with normal studies should probably occur earlier than for non-diabetic patients. Similar data exist for haemodialysis patients. ${ }^{23}$

Importantly, the prognostic power of abnormal images in diabetic patients is also preserved. Risk adjusted event-free survival in patients with mildly, and moderately to severely abnormal scans appears, however, to be worse in diabetic patients than in non-diabetic patients, and also to be worse in female diabetic patients than in male diabetic patients.

\section{AFTER CORONARY ARTERY BYPASS SURGERY AND PERCUTANEOUS INTERVENTION}

The long term effectiveness of coronary artery bypass surgery is limited by the progression of disease in native vessels and graft stenoses, with approximately $50 \%$ of saphenous vein grafts being occluded by 10 years. ${ }^{24}$ Chest pain symptoms and exercise electrocardiography are largely unhelpful for identifying those patients at risk. ${ }^{25}$ By contrast, perfusion imaging is of proven value in patients with symptoms, as well as in asymptomatic patients, more than five years after bypass 
surgery in whom it may detect silent progression of prognostically important disease. ${ }^{26}$ In asymptomatic patients less than five years after bypass surgery, annual cardiac mortality is too low to justify routine perfusion studies. ${ }^{27}$ However, where revascularisation is incomplete or technically difficult, an early postoperative perfusion scan may form a useful reference for the follow up period.

Perfusion imaging is also helpful in patients with recurrent but atypical symptoms after angioplasty; although it is best to defer imaging for six weeks as perfusion abnormalities can take this time to resolve even with a good anatomical result. Thereafter an abnormal perfusion study is predictive of adverse cardiac events. ${ }^{28}$ A normal study at any stage has excellent negative predictive value for predicting restenosis and clinical events after angioplasty. Routine perfusion imaging after angioplasty in the absence of symptoms is not common. It can, however, be justified routinely in patients with impaired left ventricular function, proximal left anterior descending coronary artery disease, suboptimal results of angioplasty, diabetes, and those with occupations requiring low coronary risk.

\section{AFTER MYOCARDIAL INFARCTION}

An important aspect of clinical management after infarction is to identify patients at high risk of further events such as reinfarction or death, and hopefully to intervene to prevent these events. Clinical indicators of high risk in the acute phase include post-infarction angina, left ventricular failure, and malignant arrhythmias, and these patients are candidates for early angiography. Patients with uncomplicated myocardial infarction are at lower risk but still require some form of stress testing to assess the presence of residual ischaemia and an assessment of left ventricular function. Symptom limited exercise electrocardiography at six weeks and an echocardiographic study are the norm but myocardial perfusion imaging offers clear advantages. Numerous studies attest to the fact that it provides superior prognostic information to exercise electrocardiography. Perfusion imaging is more sensitive in detecting inducible ischaemia, can localise ischaemia to individual coronary territories, determine infarct size, and provide a simultaneous assessment of left ventricular function when ECG gated perfusion imaging is used. Perfusion imaging coupled with vasodilator stress can be performed safely as early as three days post-infarction, before the majority of recurrent clinical events occur, and can be used to guide early discharge, ${ }^{29}$ though logistical difficulties often make this approach impracticable in the UK. Importantly, the prognostic value of myocardial perfusion imaging after infarction is as strong in patients receiving reperfusion therapy as those managed conservatively. ${ }^{30}$

Accurate determination of viable myocardium in the infarct zone in patients with resting left ventricular dysfunction allows for the selection of patients who benefit most from revascularisation strategies. Nuclear techniques include thallium rest-redistribution imaging and perfusion-flourodeoxyglucose (FDG) metabolism positron emission tomography (PET) which have almost comparable accuracy. ${ }^{31}$ The greater the extent of pre-operative viability, the better the short and long term outcomes are with respect to survival, and improvement in LVEF and heart failure symptoms. ${ }^{32}$ If, in addition to myocardial viability, inducible ischaemia is also being evaluated, then a stress injection of tracer and imaging is also required.

\section{PRE-OPERATIVE RISK STRATIFICATION}

Perfusion imaging can provide useful information about cardiac risk in patients requiring non-cardiac surgery, ${ }^{33}$ although these patients are generally at low risk and the predictive value of a normal perfusion study is greater than that of an abnormal study. A useful algorithm upon which to base the decision as to whether pre-operative risk assessment is required is found in the American College of Cardiology/ American Heart Association pre-operative evaluation guidelines $^{34}$ which integrate four main pieces of data: the urgency for surgery and its inherent cardiac risk, the patient's risk factors, and his or her exercise tolerance. Patients with only minor clinical predictors (advanced age, abnormal resting ECG, previous stroke, or uncontrolled hypertension) who require low to moderate risk surgery, are at low risk and do not require further investigation. Patients with intermediate clinical predictors (mild angina, prior infarction, treated heart failure, or diabetes) or with minor predictors and reduced exercise tolerance need further assessment before moderate or high risk surgery. Patients at high clinical risk (recent infarction or unstable angina, uncontrolled heart failure or significant arrhythmias) require investigation before any sort of surgery. Where further investigation is required the choice lies primarily between exercise electrocardiography and perfusion imaging, the latter usually being reserved for those patients with an abnormal resting ECG or inability to exercise. In general, patients identified as low risk can undergo surgery without further investigation. All other patients require aggressive medical management at the time of surgery, intervention usually being reserved for those in whom revascularisation is indicated regardless of the need for surgery.

\section{CONCLUSIONS}

Beyond diagnosis, the most valuable contribution that perfusion imaging can make to patient management is to assess the likelihood of important coronary events. Prognosis is strongly influenced by the extent and severity of inducible perfusion abnormality and this can guide the need for invasive evaluation and revascularisation. Myocardial perfusion imaging provides superior prognostic data to either clinical assessment or exercise electrocardiography and often provides incremental prognostic value even once other tests have been performed..$^{35}$

The most recent estimate of UK nuclear cardiology activity was 1.12 studies/1000 population/year, ${ }^{36}$ which is $50 \%$ lower than coronary angiographic activity for the subsequent year ${ }^{37}$ and well below the level recommended by the British Cardiac Society in 1994, as adequate to serve the needs of patients with cardiac disease in the UK. ${ }^{38}$ These figures suggest that despite overwhelming evidence that perfusion imaging provides effective risk assessment, referring physicians in the UK are not routinely using nuclear techniques for this purpose. If this were the case then perfusion imaging activity would inevitably be higher than coronary angiographic activity, probably by a factor of $2-3$. The reasons for this are multifactorial but include the fact that cardiologists prefer to use techniques in their control such as coronary angiography, continuing, although diminishing, scepticism about nuclear techniques among cardiologists, the poor quality reports provided by some centres, ${ }^{39}$ and long waiting lists for perfusion imaging. Hopefully, this series of articles and other educational initiatives will address the first of these issues; the others are for the nuclear medicine community as a whole to address.

\section{Authors' affiliations}

T M Bateman, Cardiac and Vascular Radiologic Imaging, Mid America Heart Institute, Department of Medicine, University of Missouri-Kansas City, Kansas City, Missouri, USA

E Prvulovich, Institute of Nuclear Medicine, Middlesex Hospital, London, UK

Correspondence to: Dr Elizabeth Prvulovich, Institute of Nuclear Medicine, Middlesex Hospital, Mortimer Street, London WIN 8AA, UK; I.prvulovich@nucmed.ucl.ac.uk 


\section{REFERENCES}

1 Klocke $\mathbf{F}$, et al. ACC/AHA/ASNC guidelines for the clinical use of cardiac radionuclide imaging. J Am Coll Cardiol 2003;42:1318-33

2 Hachamovitch $\mathbf{R}$, Berman DS, Kiat $\mathrm{H}$, et al. Exercise myocardial perfusion SPECT in patients without known coronary artery disease: incremental prognostic value and use in risk stratification. Circulation 1996;93:905-14.

3 Gill JB, Ruddy TD, Newell JB, et al. Prognostic importance of thallium uptake by the lungs during exercise in coronary artery disease. N Engl J Med 1987:317:1486-9.

4 Weiss AT, Berman DS, Lew AS, et al. Transient ischemic dilation of the left ventricle on stress thallium-201 scintigraphy: a marker of severe and extensive coronary artery disease. J Am Coll Cardiol 1987;9:752-9.

5 Gioia G, Milan E, Giubbini R, et al. Prognostic value of tomographic restredistribution thallium 201 imaging in medically treated patients with coronary artery disease and left ventricular dysfunction. J Nucl Cardiol 1996;3:150-6.

6 Hachamovitch R, Berman DS, Shaw $\sqcup$, et al. Incremental prognostic value of myocardial perfusion single photon emission computed tomography for the prediction of cardiac death: differential stratification for risk of cardiac death and myocardial infarction. Circulation 1998;97:535-43.

7 Sharir T, Germano G, Kang X, et al. Prediction of myocardial infarction versus cardiac death by gated myocardial perfusion SPECT: risk stratification by the amount of stress-induced ischemia and the poststress ejection fraction. J Nucl Med 2001;42:831-47.

8 Little WC, Constantinescu M, Applegate RJ, et al. Can coronary angiography predict the site of a subsequent myocardial infarction in patients with mild-tomoderate coronary artery disease? Circulation 1988;78:1157-66.

9 Ladenheim ML, Kotler TS, Pollock BH, et al. Incremental prognostic power of clinical history, exercise electrocardiography and myocardial perfusion scintigraphy in suspected coronary artery disease. Am J Cardiol 1987;59:270-7.

10 Iskandrian AS, Chae SC, Heo J, et al. Independent and incremental prognostic value of exercise single-photon emission computed tomographic (SPECT) thallium imaging in coronary artery disease. J Am Coll Cardiol 1993;22:665-70.

11 Myers J, Prakash M, Froelicher V, et al. Exercise capacity and mortality among men referred for exercise testing. N Engl J Med 2002;346:793-801.

12 Chatziioannou SN, Moore WH, Ford PV, et al. Prognostic value of myocardial perfusion imaging in patients with high exercise tolerance. Circulation 1999;99:867-72

13 Sharir T, Germano G, Kavanagh K, et al. Incremental prognostic value of post-stress left ventricular ejection fraction and volume by gated myocardial perfusion single photon emission computed tomography. Circulation 1999; 100:1035-42.

14 Johnson LL, Verdesca SA, Aude WY, et al. Postischemic stunning can affect left ventricular ejection fraction and regional wall motion on post-stress gated sestamibi tomograms. J Am Coll Cardiol 1997;30:1641-8.

15 Bateman TM, O'Keefe JH Jr, Dong VM, et al. Coronary angiography rates following stress SPECT scintigraphy. J Nucl Cardiol 1995;2:217-23.

16 Shaw LJ, Hachamovitch R, Berman DS, et al. The economic conseguences of available diagnostic and prognostic strategies for the evaluation of stable angina patients: an observational assessment of the value of precatheterization ischemia. J Am Coll Cardiol 1999;33:661-9.

17 Hachamovitch R, Berman DS, Kiat $\mathrm{H}$, et al. Effective risk stratification using exercise myocardial perfusion SPECT in women: gender-related differences in prognostic nuclear testing. J Am Coll Cardiol 1996;28:34-44.

18 Gibbons RJ, Hodge DO, Berman DS, et al. Long-term outcome of patients with intermediate-risk exercise electrocardiograms who do not have myocardial perfusion defects on radionuclide imaging. Circulation 1999;100:2140-5.

19 Wagdy HM, Hodge D, Christian TF, et al. Prognostic value of vasodilator myocardial perfusion imaging in patients with left bundle-branch block. Circulation 1998:97:1563-70.

20 O'Keefe JH, Bateman TM, Silvestri R, et al. Safety and diagnostic accuracy of adenosine thallium-201 scintigraphy in patients unable to exercise and those with left bundle branch block. Am Heart J 1992;124:614-21.
21 Navare SM, Mather JF, Fowler MS, et al. Are there differences in risk stratification of patients with known or suspected coronary disease between pharmacologic and exercise stress myocardial perfusion imaging? A metaanalysis [abstract]. J Nucl Cardiol 2002;4:S22

22 Giri S, Shaw $\amalg$, Murthy DR, et al. Impact of diabetes on the risk stratification using stress single-photon emission computed tomography myocardial perfusion imaging in patients with symptoms suggestive of coronary artery disease. Circulation 2002;105:32-40

23 Yusuf S, Sucker D, Peruse P, et al. Effect of coronary artery bypass graft surgery on survival: overview of 10 year results from randomized trials by the coronary artery bypass graft surgery realists collaboration. Lancet 1994;344:563-70

24 Dahan $M$, Viron B, Faraggi $M$, et al. Diagnostic accuracy and prognostic value of combined dipyridamole-exercise thallium imaging in haemodialysis patients. Kidney Int 1998:54:255-62.

25 Lauer MS, Lytle B, Pashkow F, et al. Prediction of death and myocardial infarction by screening with exercise thallium testing after coronary artery bypass gratting. Lancet 1998;351:615-22.

26 Nallamothu $\mathrm{N}$, Johnson $\mathrm{JH}$, Bagheri $\mathrm{B}$, et al. Utility of stress single photon emission computed tomography (SPECT) perfusion imaging in predicting outcome after coronary artery bypass grafting. Am J Cardiol 1997;80:1515-21

27 Zellweger MJ, Lewin HC, Lai S, et al. When to stress patients after coronary artery bypass surgery. J Am Coll Cardiol 2001;37:144-52.

28 Hecht HS, Shaw RE, Chin HL, et al. Silent ischemia after coronary angioplasty: evaluation of restenosis and extent of ischemia in asymptomatic patients by tomographic thallium-201 exercise imaging and comparison with symptomatic patients. J Am Coll Cardiol 1991;17:670-7.

29 Brown KA, Heller GV, Landin RS, et al. Early dipyridamole Tc- $99 \mathrm{~m}$ sestamibi single photon emission computed tomographic imaging 2 to 4 days after acute myocardial infarction predicts in-hospital and post-discharge cardiac events: comparison with submaximal exercise imaging. Circulation 1999;100:2060-6

30 Mahmarian JJ, Mahmarian AC, Marks GF, et al. Role of adenosine thallium201 tomography for defining long-term risk in patients after myocardial infarction. J Am Coll Cardiol 1995;25:1333-40.

31 Bax JJ, Wiins W, Cornel JH, et al. Accuracy of currently available techniques for prediction of functional recovery after revascularisation in patients with left ventricular dysfunction due to chronic coronary artery disease: comparison of pooled data. J Am Coll Cardiol 1997;30:1451-60.

32 DiCarli MF, Davidson M, Little R, et al. Value of metabolic imaging with positron emission tomography for evaluating prognosis in patients with coronary artery disease and left ventricular dysfunction. Am J Cardiol 1994;74:527-33.

33 L'Italien GJ, Paul S, Hendel RC, et al. Development and validation of a Bayesian model for postoperative cardiac risk assessment in cohort of 1081 vascular surgical candidates. J Am Coll Cardiol 1996;27:779-86.

34 ACC/AHA Practice Guidelines. Guidelines for perioperative cardiovascular evaluation for noncardiac surgery. Circulation 1996;93:1278-317.

35 Beller GA, Zaret B. Contributions of nuclear cardiology to diagnosis and prognosis of patients with coronary artery disease. Circulation $2001 ; 10: 1465-78$

36 Prvulovich E, Metcalfe MJ. Nuclear cardiology in the UK: activity and practice 1997. Eur J Nucl Med 2002;29:553-8.

37 Maier W, Windecker S, Lablanche JM, Muhlberger V, Wiins W, Meier B on behalf of the working Group Coronary Circulation of the European Society of Cardiology. The European Registry of cardiac catheter interventions 1996. Eur Heart J 2001;22:567-73.

38 British Cardiac Society. Council statement on the demand and need for cardiac services and the development of a waiting list strategy for cardiac disease. London: British Cardiac Society, July 1994.

39 Prvulovich E, Jarritt PH, Vivian GC, et al. Quality assurance in myocardial perfusion tomography: a collaborative BNCS/BNMS audit programme. Nucl Med Comm 1998;19:831-8. 